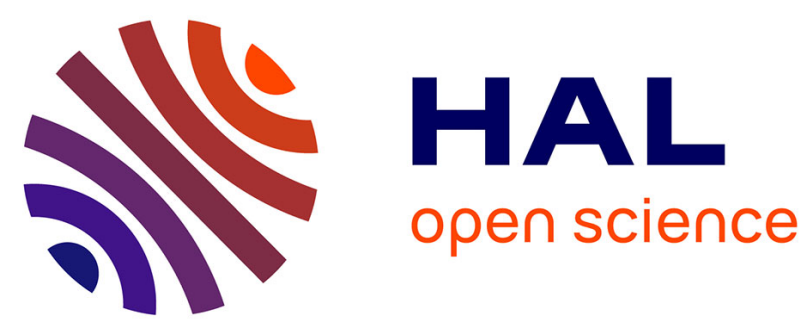

\title{
Identification of electrostatically actuated mems models from real measurement data
}

\author{
Céline Casenave, Emmanuel Montseny, Henri Camon
}

\section{To cite this version:}

Céline Casenave, Emmanuel Montseny, Henri Camon. Identification of electrostatically actuated mems models from real measurement data. 15th IFAC Symposium on System Identification, SYSID 2009, Jul 2009, Saint-Malo, France. pp.1738-1743, 10.3182/20090706-3-FR-2004.00289 . hal-01061510

\author{
HAL Id: hal-01061510 \\ https://hal.inria.fr/hal-01061510
}

Submitted on 7 Sep 2014

HAL is a multi-disciplinary open access archive for the deposit and dissemination of scientific research documents, whether they are published or not. The documents may come from teaching and research institutions in France or abroad, or from public or private research centers.
L'archive ouverte pluridisciplinaire HAL, est destinée au dépôt et à la diffusion de documents scientifiques de niveau recherche, publiés ou non, émanant des établissements d'enseignement et de recherche français ou étrangers, des laboratoires publics ou privés. 


\title{
Identification of Electrostatically Actuated MEMS Models from Real Measurement Data
}

\author{
C. Casenave*, E. Montseny*, H. Camon* \\ * LAAS-CNRS, Université de Toulouse \\ 7 av. du Colonel Roche, 31077 Toulouse cedex 4, France. \\ e-mail: casenave@laas.fr, emontseny@laas.fr, camon@laas.fr
}

\begin{abstract}
This paper focuses on the identification of nonlinear dynamic models for physical systems such as Micro-Electro-Mechanical Systems (MEMS) from measurement data. The proposed approach consists in transforming, by means of suitable global operations, the specific input-output differential physical model of the system elaborated from physical analysis, in such a way that we get a new equivalent model formulation specifically adapted to the identification problem. Thanks to the equivalence of the dynamic model and the derived identification problem, the so-identified model remains of continuous-time type, with a clear physical meaning of any of its components, which is not the case when using, for example, black-boxes approaches. The method is implemented on real measurement data from a physical system.
\end{abstract}

Keywords: Dynamic models, parameters identification, nonlinear models, least-squares identification.

\section{INTRODUCTION}

This paper focuses on the identification of nonlinear dynamic models for physical systems such as MEMS from measurement data. Due to their very small size, many parameters of those systems cannot be accurately known; in this case, identification process can be the only way to get reliable models, useful for example for control (Zhu (2007); Liao (2004); Sane (2005); Daqaq (2006); Bryzek (2003)) or even dimensioning purposes. Several informations and techniques about identification of dynamic systems will be found in Ljung (1999) and Garnier (2004).

In this paper, we identify parameters and functional components of a nonlinear dynamic model presenting a bifurcation point. The proposed approach consists in transforming, by means of suitable global operations, the specific input-output differential physical model of the system elaborated from physical analysis, in such a way that we get a new equivalent model formulation well adapted to the identification problem. By using spline interpolations, we establish a time-continuous identification problem which will be solved by classical least-square methods, with the only shortcoming of a small and easy to suppress identification bias. Moreover, thanks to the equivalence of the dynamic model and the derived identification problem, the so-identified model remains of continuous-time type, with a clear physical meaning of any of its components, which is not the case when using, for example, black-boxes approaches.

The paper is organized as follows. We present the physical system and its dynamic model, and then state the associated identification problem. After that we express the solution of this problem in terms of operatorial pseudoinversion and describe how to solve the problem numeri- cally from a discrete set of measurement data. Finally, we implement the method on real measurement data.

\section{THE PHYSICAL SYSTEM UNDER CONSIDERATION}

The system under consideration is an electrostatically actuated micromirror ${ }^{1}$ (cf. Fig. 1). It is composed in two assembled parts. The upper one is a thin plate, the mirror, linked to a thick external rigid frame by two thin and narrow arms, the springs. This part is tailored in the same microcrystalline silicon layer of a SOI (Silicon On Insulator) wafer. The lower one comprises a balance-knifeedge with two electrodes distributed on both sides of it. The two parts are assembled in such a manner that the axis of the springs and balance-knife-edge are identical. So the electrodes are located underneath the mirror inducing its rotation (left or right) when a voltage $V$ is applied.

The development of those micro-mirrors has been conducted with Tronics Microsystems (France), a manufacturer of custom MEMS components. Several configurations of the electrode can be envisaged, namely the case where electrodes are flat or inclined (cf. Fig. 2 and 3 for flat electrodes configuration). Due to the fabrication process, essentially the gluing process of the two parts, it is realistic to consider that the physical structure is significantly different from the ideal one, and so do the associated dynamic model.

In the sequel the physical limit angle the mirror can reach is denoted $\alpha$, whereas $\theta(t)$ denotes the angle of the mirror at a given time (see Fig. 3).

1 The picture is published with courtesy of Tronics Microsystems (France). 


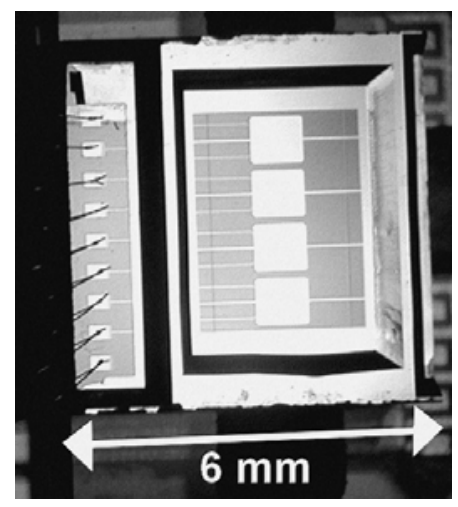

Fig. 1. View of the physical system

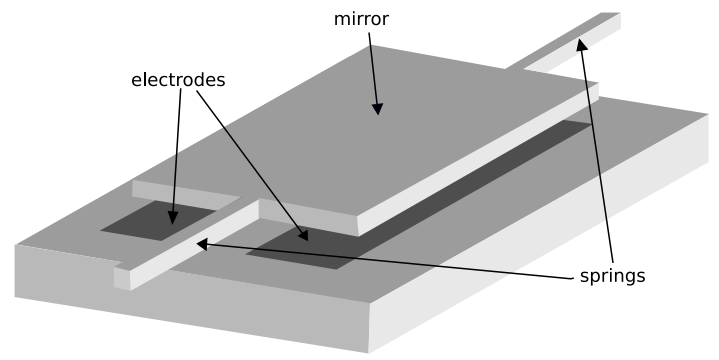

Fig. 2. Schematic drawing of the MEMS with flat electrodes

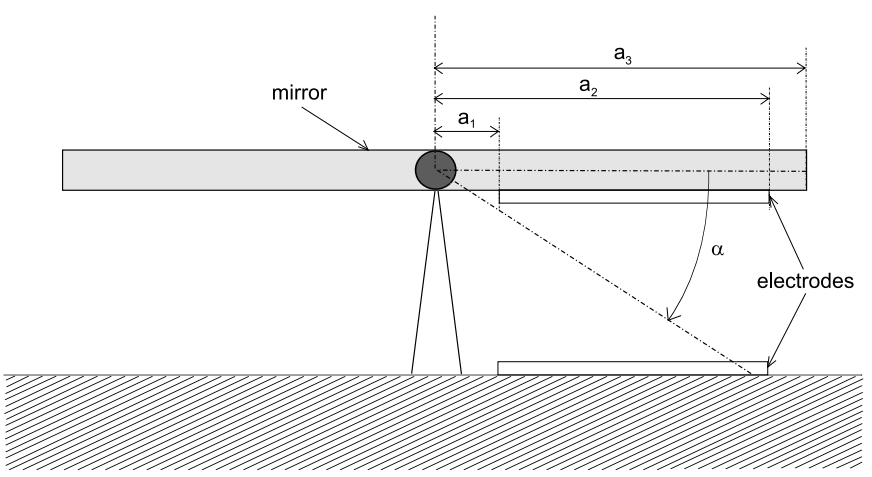

Fig. 3. Cross section of the MEMS with flat electrodes

The dynamic model of the system is obtained by application of the fundamental principle of dynamics:

$$
\begin{aligned}
& I \ddot{\theta}+\left(\mu_{0}+v(\theta)\right) \dot{\theta}+K \theta=V^{2} k(\theta), \\
& \text { with the constraint }:|\theta| \leqslant|\alpha|,
\end{aligned}
$$

where $I$ is the inertia moment of the system, $V^{2} k(\theta)$ is the electrostatic moment, $K \theta$ the spring moment, and $\left(\mu_{0}+v(\theta)\right) \dot{\theta}$ the viscous friction moment. The system (1) of input $V$ and output $\theta$, is completed by the initial conditions:

$$
\theta(0)=0 \text { and } \dot{\theta}(0)=0 .
$$

Remark 1. In the model, $\theta$ is a negative angle.

The expression of the terms $v(\theta)$ and $k(\theta)$ involved in model (1) depends on the configuration of the physical system (flat or inclined electrodes), its geometry, the materials used etc. The analysis of this dynamic system (Casenave (2008)) reveals the existence of a threshold voltage denoted $V_{\text {pullin }}^{\text {dyn }}$, below which the mirror stabilizes to an angle $\theta_{\text {stab }}(V)$ (see Fig. 4a), whereas beyond this voltage the mirror switches (see Fig. 4b).

We focus in this paper on the problem of identification of model (1) (namely identification of inertia moment $I$, electrostatic moment function $k$, etc.) from real measurement data. This problem is tricky for several reasons: the model is nonlinear, and presents a bifurcation point that separate two different dynamic behaviors of the system. Moreover, inertia identification is difficult because its contribution is often (but not always) dominated by viscosity terms.

\section{FORMULATION AND RESOLUTION OF THE IDENTIFICATION PROBLEM}

In this section, we state and study the identification problem under consideration, whose aim is to estimate the parameters of model $(1,3)$ from measurements of trajectories $\theta$.

\subsection{Preliminaries for the identification problem}

As said previously, the expression of electrostatic and viscous friction moments functions $k$ and $v$ depends on the physical system, and in general it may not be accurately known. We identify the two functions under the form:

$$
\begin{aligned}
& k(\theta)=\sum_{l} c_{l} \mathbf{k}_{l}(\theta) \\
& v(\theta)=\sum_{q} \mu_{q} \mathbf{v}_{q}(\theta),
\end{aligned}
$$

where the parameters to be identified are the real coefficients $c_{l}$ and $\mu_{q}$. From the theoretical point of view, these sums can be finite or not and we implicitly refer to functional Hilbert spaces generated by $\mathbf{k}_{l}$ and $\mathbf{v}_{q}$, to which belong $k$ and $v$. In the sequel, we will use the notations:

$$
\begin{aligned}
c & :=\left(c_{1}, c_{2}, \ldots\right)^{T} \in \ell^{2}, \mathbf{k}(\theta):=\left(\mathbf{k}_{1}(\theta), \mathbf{k}_{2}(\theta), \ldots\right), \\
\mu & :=\left(\mu_{1}, \mu_{2}, \ldots\right)^{T} \in \ell^{2}, \mathbf{v}(\theta):=\left(\mathbf{v}_{1}(\theta), \mathbf{v}_{2}(\theta), \ldots\right) .
\end{aligned}
$$

So we can write in a condensed form:

$$
k(\theta)=\mathbf{k}(\theta) c, v(\theta)=\mathbf{v}(\theta) \mu .
$$

Then, we consider the possibility of transforming the model (1) in an equivalent manner by composition with any causal convolution operator $\mathcal{H}:=H\left(\partial_{t}\right)$ with impulse response $h=\mathcal{L}^{-1} H$ :

$$
\begin{gathered}
\left(\mathcal{H} \circ \partial_{t}^{2}\right) \theta I+\left(\mathcal{H} \circ \partial_{t}\right) \theta \mu_{0}+\mathcal{H}\left(\mathbf{v}(\theta) \partial_{t} \theta\right) \mu+(\mathcal{H} \theta) K= \\
V^{2} \mathcal{H}(\mathbf{k}(\theta)) c .
\end{gathered}
$$

To avoid the amplification of noise measurement by operators $\partial_{t}$ and $\partial_{t}^{2}$, the operator $H\left(\partial_{t}\right)$ has to be chosen in such a way that high frequencies are attenuated, that is $|H(i \omega)|^{2} \underset{H . F}{\sim} \frac{1}{\omega^{2}}$, without amplifying low ones $H(i \omega)^{2} \underset{\text { L.F }}{\sim}$ 1: basically, it behaves like a second order low-pass filter.

Finally, it is obvious that, in model (9), the parameters can only be identified up to a multiplicative constant. Then, we consider that $\mu_{0}=1$ and denote:

$$
\lambda:=(I, \mu, K, c)^{T} \in E:=\mathbb{R} \times \ell^{2} \times \mathbb{R} \times \ell^{2}
$$


the vector of parameters to be identified. The exact (but unknown) value of $\lambda$ is denoted $\lambda_{0}$. We can then rewrite (9) under the synthetic form, used for identification purposes:

$$
\mathcal{A}_{\theta} \lambda=b_{\theta},
$$

where $\mathcal{H}_{1}:=\mathcal{H} \circ \partial_{t}, \mathcal{H}_{2}:=\mathcal{H} \circ \partial_{t}^{2}, b_{\theta}:=-\mathcal{H}_{1} \theta \in$ $L^{2}\left(0, T ; \mathbb{R}^{J}\right)$ and $\mathcal{A}_{\theta}: E \longrightarrow L^{2}\left(0, T ; \mathbb{R}^{J}\right)$ is the matrix operator defined by:

$$
\mathcal{A}_{\theta}=\left[\begin{array}{llll}
\mathcal{H}_{2} \theta & \mathcal{H}\left(\mathbf{v}(\theta) \partial_{t} \theta\right) & \mathcal{H} \theta & -V^{2} \mathcal{H}(\mathbf{k}(\theta))
\end{array}\right] .
$$

Remark 1. In practice, we will manipulate multiple trajectories $\theta^{j}, j=1: J$ obtained with different input voltages $V^{j}$ (the associated initial conditions are all taken equal to 0 ). Then, model (9) can be extended to the general case of multiple trajectories by considering:

$$
\begin{aligned}
& \theta=\left(\theta^{1}, \ldots, \theta^{J}\right)^{T}, \partial_{t} \theta=\left(\partial_{t} \theta^{1}, \ldots, \partial_{t} \theta^{J}\right)^{T} \\
& \mathbf{v}(\theta)=\left[\begin{array}{ccc}
\mathbf{v}_{1}\left(\theta^{1}\right) & \mathbf{v}_{2}\left(\theta^{1}\right) & \cdots \\
\vdots & \vdots & \\
\mathbf{v}_{1}\left(\theta^{J}\right) & \mathbf{v}_{2}\left(\theta^{J}\right) & \cdots
\end{array}\right], \\
& \mathbf{k}(\theta)=\left[\begin{array}{ccc}
\mathbf{k}_{1}\left(\theta^{1}\right) & \mathbf{k}_{2}\left(\theta^{1}\right) & \cdots \\
\vdots & \vdots & \\
\mathbf{k}_{1}\left(\theta^{J}\right) & \mathbf{k}_{2}\left(\theta^{J}\right) & \ldots
\end{array}\right] \text {, } \\
& \text { and } V=\left[\begin{array}{ccc}
V^{1} & & 0 \\
& \ddots & \\
0 & & V^{J}
\end{array}\right] \text {. }
\end{aligned}
$$

\subsection{Formulation and resolution of the identification problem}

The aim is to estimate $\lambda$ from experimental data of the form: $\theta_{\mathrm{m}}=\theta+\eta$, with $\eta$ some additive measurement noise such that, for simplicity, $\mathbb{E}(\eta)=0$. We then write the identification problem under the following form, taking advantage from the linearity of (11) with respect to parameter $\lambda$ :

$$
\min _{\lambda \in E}\left\|\mathcal{A}_{\theta_{\mathrm{m}}} \lambda-b_{\theta_{\mathrm{m}}}\right\|_{L^{2}\left(0, T ; \mathbb{R}^{J}\right)}^{2}
$$

Its solution $\lambda^{*}$ is formally obtained by:

$$
\lambda^{*}=\mathcal{A}_{\theta_{\mathrm{m}}}^{\dagger} b_{\theta_{\mathrm{m}}}
$$

where $\mathcal{A}_{\theta_{\mathrm{m}}}^{\dagger}$ designates the pseudo-inverse of operator $\mathcal{A}_{\theta_{\mathrm{m}}}$, defined by (Ben-Israel (2003)):

$$
\mathcal{A}_{\theta_{\mathrm{m}}}^{\dagger}=\left(\mathcal{A}_{\theta_{\mathrm{m}}}^{*} \mathcal{A}_{\theta_{\mathrm{m}}}\right)^{-1} \mathcal{A}_{\theta_{\mathrm{m}}}^{*} n
$$

with $\mathcal{A}_{\theta_{\mathrm{m}}}^{*}$ the adjoint operator of $\mathcal{A}_{\theta_{\mathrm{m}}}$.

In particular, we have from (11): $A_{\theta} \lambda_{0}=b_{\theta}$; so, the estimator $\lambda^{*}$ is exact when the data are unoised:

$$
A_{\theta}^{\dagger} b_{\theta}=\lambda_{0} \text {. }
$$

Because operator $\mathcal{A}_{\theta_{\mathrm{m}}}$ depends on the measurement noise, the solution $\lambda^{*}$ of (14) will be a biased estimator of $\lambda_{0}$. However, an efficient iterative procedure can be used to reduce the bias and thus increase the identification accuracy (Casenave (2008)).

\section{NUMERICAL FORMULATION}

In this section, we show how to numerically solve the continuous time (infinite dimensional) identification problem introduced above.

\subsection{Discrete data interpolation}

In practice we only have access to discrete trajectories, that is several sets of data $\left\{\theta_{\mathrm{m}}^{j, k}\right\}_{k=1: K}, j=1: J$, where $\theta_{\mathrm{m}}^{j, k}$ is a measurement of $\theta\left(t_{k}, V_{j}\right)$ with $t_{k+1}=t_{k}+$ $\Delta t$. To go back to a continuous-time formulation, we use cubic spline interpolations which allow the analytical computation of the involved quantities (such as $A_{m}$ and $\mathrm{b}_{\mathrm{m}}$ introduced later).

In the sequel we will denote $\theta_{\mathrm{m}}^{j}: t \mapsto \theta_{\mathrm{m}}^{j}(t)$ the cubic spline interpolation of the set $\left\{\left(t_{k}, \theta_{\mathrm{m}}^{j, k}\right)\right\}_{k}$. We have:

$$
\theta_{\mathrm{m}}^{j}=\theta^{j}+\eta
$$

where $\theta^{j}$ is the exact (unknown) trajectory solution of (9) (with $V=V^{j}$ ), and $\eta$ is an additional noise resulting both from measurement errors and interpolation ones. In the same way, cubic spline interpolation will be made on the sets $\left\{\left(t_{k}, \mathbf{v}_{q}\left(\theta_{\mathrm{m}}^{j, k}\right) \partial_{t} \theta_{\mathrm{m}}^{j, k}\right)\right\}_{k}$ and $\left\{\left(t_{k}, \mathbf{k}_{l}\left(\theta_{\mathrm{m}}^{j, k}\right)\right)\right\}_{k}$ to get a continuous-time identification problem of the form (14), whose numerical solution is obtained as described here after.

\subsection{Numerical resolution of (14)}

To numerically solve the minimisation problem (14) which is a priori of infinite dimension, we first have to replace the infinite sums (8) by truncations at finite orders $L$ and $Q$ :

$$
\mathbf{k}\left(\theta^{j}\right) c \simeq \sum_{l=1}^{L} c_{l} \mathbf{k}_{l}\left(\theta^{j}\right) \text { and } \mathbf{v}\left(\theta^{j}\right) \mu \simeq \sum_{q=1}^{Q} \mu_{q} \mathbf{v}_{q}\left(\theta^{j}\right)
$$

In the sequel, $\mathbf{k}(\theta)$ and $\mathbf{v}(\theta)$ will now designate the respective finitedimensional matrices:

$$
\left[\begin{array}{ccc}
\mathbf{v}_{1}\left(\theta^{1}\right) & \cdots & \mathbf{v}_{Q}\left(\theta^{1}\right) \\
\vdots & & \vdots \\
\mathbf{v}_{1}\left(\theta^{J}\right) & \cdots & \mathbf{v}_{Q}\left(\theta^{J}\right)
\end{array}\right] \text { and }\left[\begin{array}{ccc}
\mathbf{k}_{1}\left(\theta^{1}\right) & \cdots & \mathbf{k}_{L}\left(\theta^{1}\right) \\
\vdots & & \vdots \\
\mathbf{k}_{1}\left(\theta^{J}\right) & \cdots & \mathbf{k}_{L}\left(\theta^{J}\right)
\end{array}\right] \text {. }
$$

We then discretize the problem (14). Let $\left\{t_{n}\right\}_{n=1: N}$ a time discretization. For any $t_{n}$ we have:

$$
\left(\mathcal{A}_{\theta} \lambda\right)\left(t_{n}\right)=b_{\theta}\left(t_{n}\right) \text {. }
$$

Let $\mathrm{A}_{\mathrm{m}}: \mathbb{R} \times \mathbb{R}^{Q} \times \mathbb{R} \times \mathbb{R}^{L} \longrightarrow\left(\mathbb{R}^{J}\right)^{N}$ the block-matrix:

$$
\mathrm{A}_{\mathrm{m}}:=\left[\begin{array}{c}
\mathrm{A}_{\theta_{m}}\left(t_{1}\right) \\
\vdots \\
\mathrm{A}_{\theta_{m}}\left(t_{N}\right)
\end{array}\right]
$$

where $\mathrm{A}_{\theta_{\mathrm{m}}}\left(t_{n}\right): \mathbb{R} \times \mathbb{R}^{Q} \times \mathbb{R} \times \mathbb{R}^{L} \longrightarrow \mathbb{R}^{J}$ is the matrix defined by:

$$
\begin{aligned}
\mathrm{A}_{\theta_{\mathrm{m}}}\left(t_{n}\right):=[ & \left(\mathcal{H}_{2} \theta_{\mathrm{m}}\right)\left(t_{n}\right)\left(\mathcal{H}\left(\mathbf{v}\left(\theta_{\mathrm{m}}\right) \partial_{t} \theta_{\mathrm{m}}\right)\right)\left(t_{n}\right)\left(\mathcal{H} \theta_{\mathrm{m}}\right)\left(t_{n}\right) \\
& \left.-V^{2}\left(t_{n}\right)\left(\mathcal{H}\left(\mathbf{k}\left(\theta_{\mathrm{m}}\right)\right)\right)\left(t_{n}\right)\right] .
\end{aligned}
$$

Let also define:

$$
\mathrm{b}_{\mathrm{m}}:=\left[\begin{array}{c}
-\left(\mathcal{H}_{1} \theta_{\mathrm{m}}\right)\left(t_{1}\right) \\
\vdots \\
-\left(\mathcal{H}_{1} \theta_{\mathrm{m}}\right)\left(t_{N}\right)
\end{array}\right] \in\left(\mathbb{R}^{J}\right)^{N}
$$

Now, we can write the numerical formulation of the identification problem (14) (with possible reconditioning small parameter $\varepsilon \geqslant 0)$ :

$$
\min _{\lambda}\left\{\left\|A_{m} \lambda-b_{m}\right\|^{2}+\varepsilon\|\lambda\|^{2}\right\},
$$


whose solution is classically given by:

$$
\lambda^{*}=\left(\mathrm{A}_{\mathrm{m}}^{T} \mathrm{~A}_{\mathrm{m}}+\varepsilon I\right)^{-1} \mathrm{~A}_{\mathrm{m}}^{T} \mathrm{~b}_{\mathrm{m}}
$$

Remark 2. The dimension of the square matrix $\mathrm{A}_{\mathrm{m}}^{T} \mathrm{~A}_{\mathrm{m}}+\varepsilon I$ is rather small (equals to the number of coefficients to be identified).

\section{APPLICATION TO REAL MEASUREMENT DATA}

In Casenave (2008) the method was first validated on simulated data. The results obtained were good: all parameters of the model (inertia, stiffness, electrostatic moment function etc.) were accurately identified (error less than $1 \%$ ), and identified curves perfectly fit the data. We implement in this section the identification method on real data, measured on a MEMS like described in section 2.

\subsection{Physical system and measurement data}

The MEMS under consideration has flat electrodes as described in Fig. 3, with $\alpha=-0.0215 \mathrm{rad}, a_{1}=150 \mu \mathrm{m}$, $a_{2}=280 \mu \mathrm{m}$ and $a_{3}=300 \mu \mathrm{m}$. The width of the mirror is equal to $600 \mu \mathrm{m}$ and its thickness is equal to $10 \mu \mathrm{m}$ (Camon (2008)).

Data available for identification are composed of $J=$ 36 sets of data $\left\{\theta_{\mathrm{m}}^{j, k}\right\}_{k=1: K}$ with $K=501$, obtained by sampling at frequency $200 \mathrm{kHz}$ during $10 \mathrm{~ms}$ of 36 measured trajectories of $\theta$. The associated inputs voltages are described here-after. During the first $5 \mathrm{~ms}$, the voltage between electrodes is fixed at a constant value $V_{j}$; thus, the system either switches or stabilizes at $\theta_{\infty}^{j}<\alpha$. During the next $5 \mathrm{~ms}$, the two electrodes are at the same potential: the system then asymptotically returns to its rest position (say $\theta=0, \dot{\theta}=0)$. The 36 voltage values $V_{j}$ are distributed from $6.085 \mathrm{~V}$ to $97.36 \mathrm{~V}$, with $V_{j}<V_{j+1}$.

Two examples of data trajectories are given in Fig. $4 \mathrm{a}$ and 4b. Note, that only the parts of data which are compatible with the model are used for identification. For example, in Fig. $4 \mathrm{~b}$, the saturation zone $[0.0045 \mathrm{~s}, 0.005 \mathrm{~s}]$ where $\theta=\alpha$ is not relevant (saturation).

\subsection{Identification model}

The identification model considered in the sequel is of the form (9) with:

- $k(\theta)$ identified under the form (19) with $L=6$ and:

$$
\mathbf{k}_{l}(\theta)=\theta^{l-1}, l=1: 6 \text {. }
$$

Indeed, we suppose the electrostatic moment can be accurately described (in the domain $[0, \alpha]$ in which $\theta$ evolves) and identified by means of a fifth order polynomial.

Remark 3. In fact, a simplified physical analysis leads to the expression: $M_{e}(\theta, V) \propto V^{2} g(\theta)$ with (Camon (2000)):

$$
g(\theta)=\frac{1}{\theta^{2}}\left[\frac{1}{1-\frac{a_{2}}{a_{3}} \frac{\theta}{\alpha}}-\frac{1}{1-\frac{a 1}{a_{3}} \frac{\theta}{\alpha}}+\ln \left(\frac{1-\frac{a_{2}}{a_{3}} \frac{\theta}{\alpha}}{1-\frac{a 1}{a_{3}} \frac{\theta}{\alpha}}\right)\right] .
$$

If this expression would be reliable, then we could identify the electrostatic moment under this form by simply considering (19) with $L=1$ and $\mathbf{k}_{1}=g$. However, due to several approximations in the physical analysis, the function (28) in fact reveals itself ill adapted for large values of $\theta$.

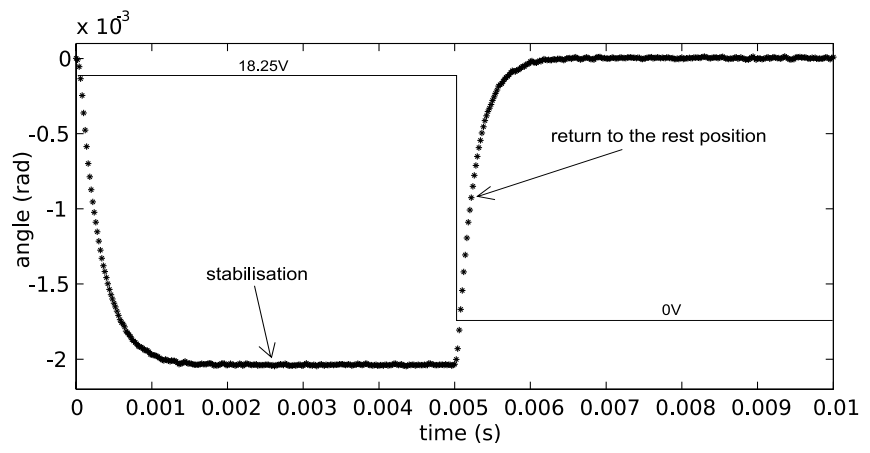

(a) $V=18.25 \mathrm{~V}<V_{\text {pullin }}^{\text {dyn }}$

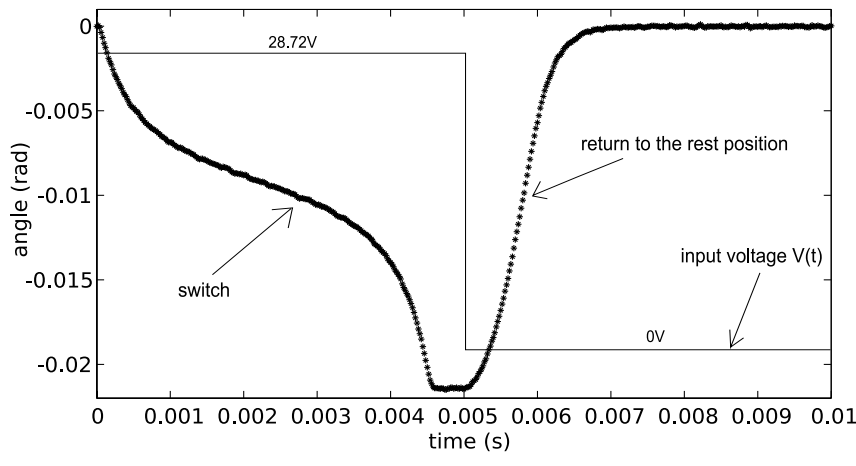

(b) $V=28.72 \mathrm{~V}>V_{\text {pullin }}^{\text {dyn }}$

Fig. 4. Data $\theta_{\mathrm{m}}^{j, k}$ for different values of $V_{j}$

$v(\theta)$ identified under the form (19) with $Q=1$ and:

$$
\mathbf{v}^{1}(\theta)=\theta^{3} .
$$

Indeed, a linear viscosity term $\mu_{0} \dot{\theta}$ is not sufficient to correctly describe the viscous moment in its whole, namely when both $|\theta|$ and $|\dot{\theta}|$ become large.

- for the prefiltering operator $\mathcal{H}$, a simple second order low-pass filter with transfer function:

$$
H(p)=\frac{\sigma^{2}}{(p+\sigma)^{2}},
$$

with $\sigma=9.0 \times 10^{3}>0$ (the cutoff frequency) chosen in such a way that $\left\|\mathcal{A}_{\theta_{\mathrm{m}}} \lambda-b_{\theta_{\mathrm{m}}}\right\|^{2}$ be "small". We so have:

$$
\left(H\left(\partial_{t}\right) u\right)(t)=\sigma^{2} \int_{0}^{t}(t-s) e^{-\sigma(t-s)} u(s) d s
$$

\subsection{Identification results}

The algorithm described in section 4 has been implemented on the sets of trajectories $\left\{\theta_{\mathrm{m}}^{j, k}\right\}, j=8: 20$, associated with $V_{j} \in[18.25 \mathrm{~V}, 31.64 \mathrm{~V}]$ : only significant data of the first $5 \mathrm{~ms}$ are considered. The system being initially at rest, the initial conditions are equal to 0 . We obtain the following identified parameters: 


$$
\begin{aligned}
& I^{*}=1.944 \times 10^{-5}, K^{*}=3.874 \times 10^{3} \\
& \mu_{1}^{*}=-5.061 \times 10^{5}, \quad c^{*}=\left[\begin{array}{c}
0.0208 \\
-1.0252 \\
217.228 \\
9.950 \times 10^{3} \\
3.0043 \times 10^{5} \\
-4.2838 \times 10^{7}
\end{array}\right] .
\end{aligned}
$$

As said previously, the parameters are identified up to the multiplicative constant $\mu_{0}$. To deduce the physical parameters from the identified quantities, we must know (or compute) the value of one parameter, from which we can deduce all the others. For example, we compute here the physical value $\tilde{I}$ of inertia moment from the dimensions of the MEMS (see Fig. 3$)$ and the density of silicon $(2.33 \times$ $10^{3} \mathrm{~kg} / \mathrm{m}^{3}$ ), and then deduce other physical quantities:

$$
\begin{aligned}
\tilde{I} & =2.693 \times 10^{-16} \mathrm{~N} \mathrm{~m} \mathrm{~s}^{2} / \mathrm{rad},(\text { computed) } \\
\tilde{\mu}_{0} & =\frac{\tilde{I}}{I^{*}} \mu_{0}=1.385 \times 10^{-11} \mathrm{~N} \mathrm{~m} \mathrm{~s} / \mathrm{rad} \\
\tilde{K} & =\frac{\tilde{I}}{I^{*}} K^{*}=5.366 \times 10^{-8} \mathrm{~N} \mathrm{~m} / \mathrm{rad}
\end{aligned}
$$

and the same for $\mu_{1}^{*}$ and $c^{*}$.

In order to globally estimate the identification quality, we consider the quantity:

$$
\mathrm{E}=\frac{\sum_{j, k}\left|\theta^{j *}(k \Delta t)-\theta_{\mathrm{m}}^{j, k}\right|}{\sum_{j, k}\left|\theta_{\mathrm{m}}^{j, k}\right|} \simeq \frac{\sum_{j} \int_{0}^{K \Delta t}\left|\theta^{j *}(t)-\theta_{\mathrm{m}}^{j}(t)\right|}{\sum_{j} \int_{0}^{K \Delta t}\left|\theta_{\mathrm{m}}^{j}(t)\right|}
$$

where $\theta^{j *}$ is the trajectory of $\theta$ obtained by simulation of the identified model for an input voltage equal to $V_{j}$. This quantity represents the cumulated relative error on all the trajectories used for the identification. In the same way, we will denote $\mathrm{E}_{\mathrm{g}}$ the cumulated relative error extended to all the available measurement trajectories (thus including prediction errors). We obtain:

$$
\mathrm{E}=0.0071 \text { and } \mathrm{E}_{\mathrm{g}}=0.0095,
$$

which highlight the good precision of the identification process. In Fig. 5 we can see some examples of trajectories obtained with the identified model for $V_{10}=20.69 \mathrm{~V}<$ $V_{\text {pullin }}^{\text {dyn }}<V_{18}=29.82 \mathrm{~V}$. Those trajectories fit well the measured data. As a validation test, we show here after the results obtained in predictive situation. We give in Fig. 6 some data which has not been used for identification and their associated trajectories predicted by the identified model. Here again, all the trajectories fit measured data with a high accuracy. Note that the voltage value $V_{\max }=$ $V_{36}=97.36 \mathrm{~V}$ we used for prediction results is far from the ones used for identification (whose larger value is $31.64 \mathrm{~V}$ ): that means there is a ratio almost equal to 10 between the input $V^{2}$ of predicted curves and this of curves used for identification. In such a situation, significant identification errors on the system parameters would generate large prediction errors; in our case, the predictive results remain good and confirm the identification accuracy.

Finally, the identified electrostatic function $k$ is given in Fig. 7 and compared with physical approximation of the form (28), which clearly reveals itself inadequate for deviations of $\theta$ close to $\alpha$. As an illustration, we can see in Fig. 8 an example of simulated trajectory when the

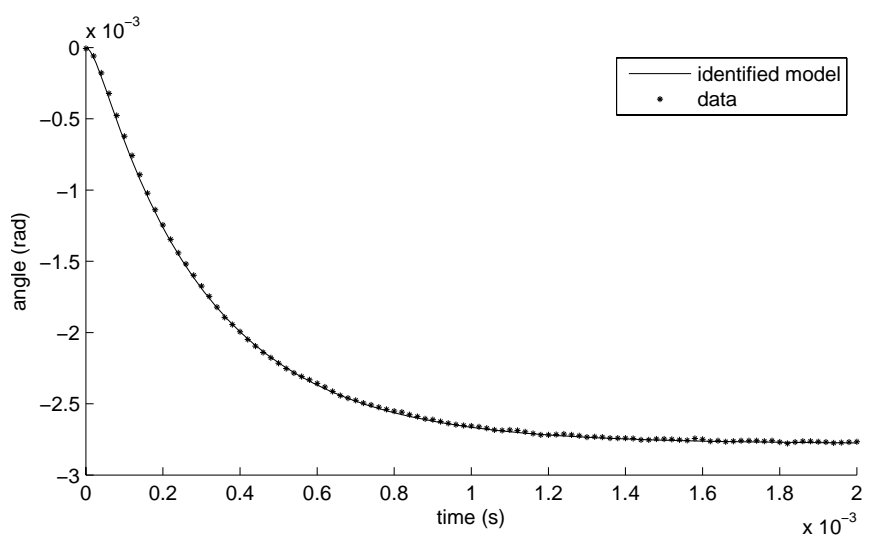

(a) $V=20.69 \mathrm{~V}$

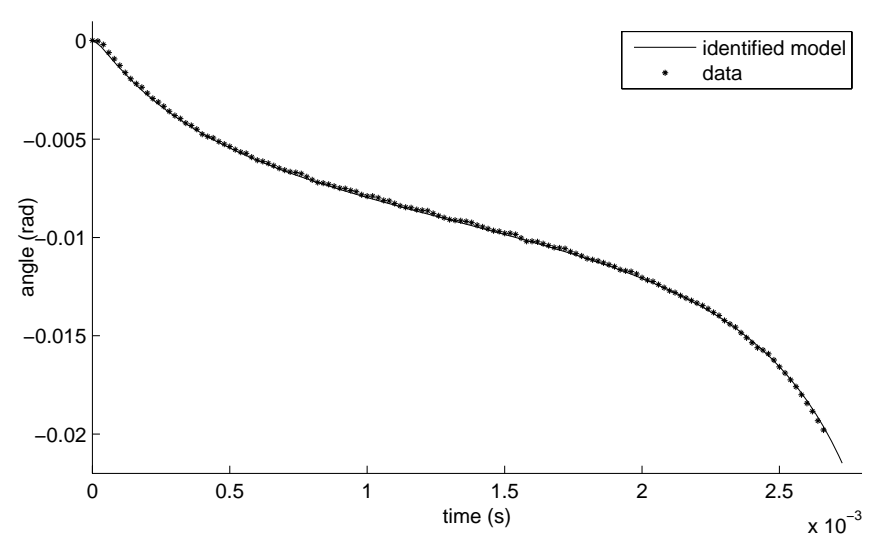

(b) $V=29.82 \mathrm{~V}$

Fig. 5. Measured trajectories and the associated $\theta$ simulated from the identified model for two values of $V$

function $k$ is under the form (28): the model does not fit with the data, contrary to the the case when $k$ is fully identified (see Fig. 5(b)).

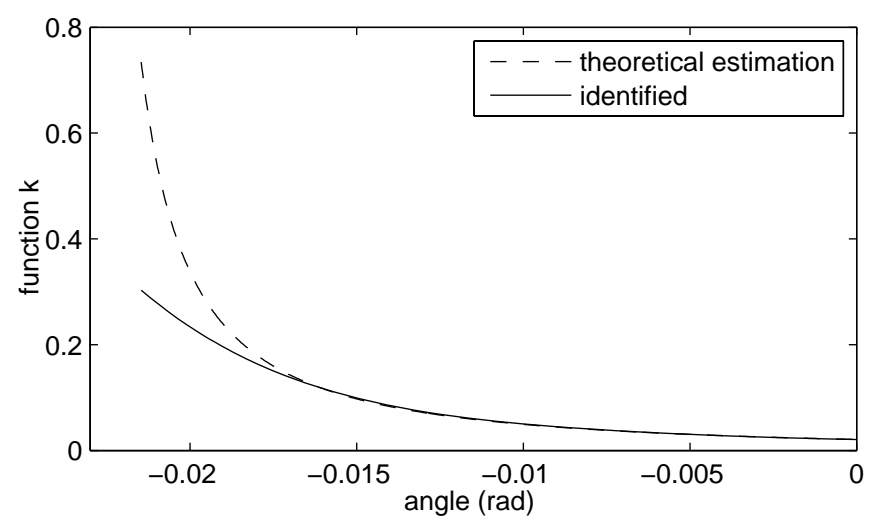

Fig. 7. The identified $k(\theta)$ and its theoretical estimation (28).

\section{CONCLUSION}

In this paper, we proposed an identification method for a nonlinear dynamic model of electrostatically actuated MEMS, using operatorial prefiltering to take advantage from the linearity with respect to the parameters to be identified. This method is applied to real measurement 


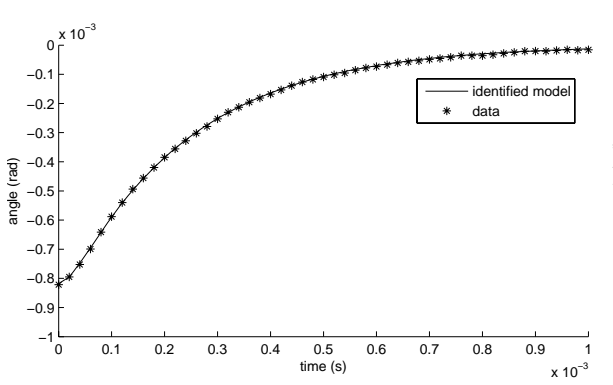

(a) return trajectory with $V=12.17 \mathrm{~V}$

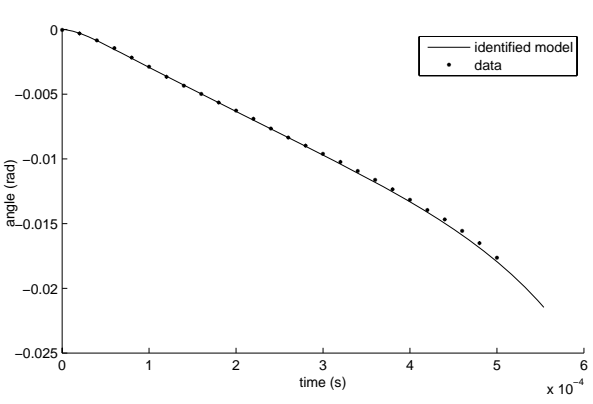

(b) $V=42.60 \mathrm{~V}$

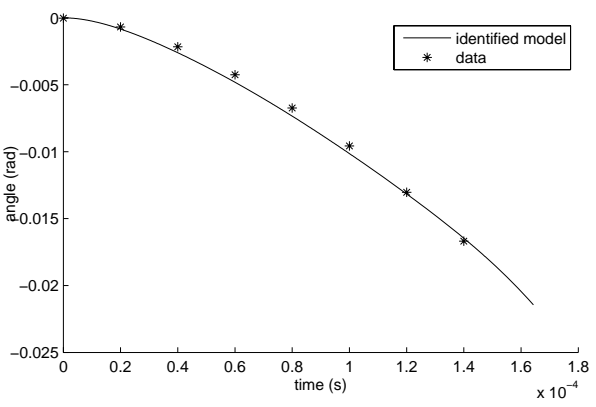

(c) $V=97.36 \mathrm{~V}$.

Fig. 6. Measured trajectories and the associated $\theta$ predicted by the identified model for different values of $V$

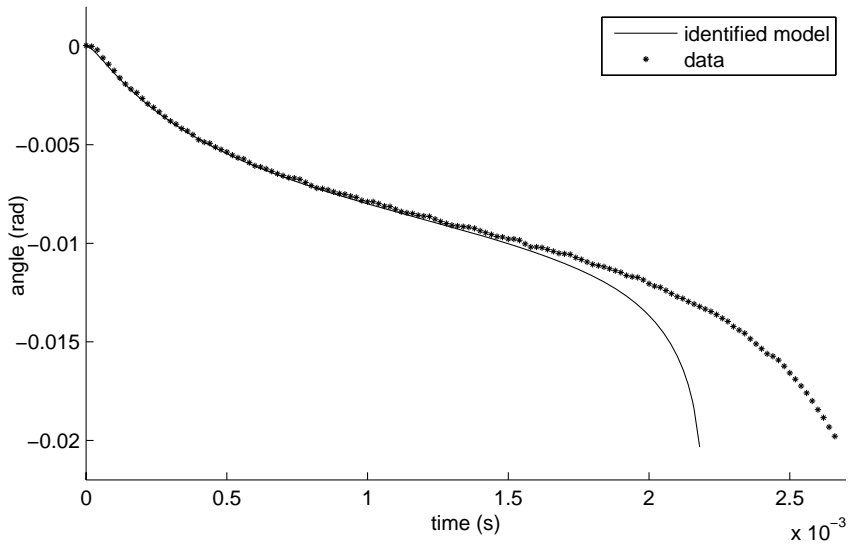

Fig. 8. Measured trajectory and the associated simulated $\theta$ when $k(\theta)$ is identified under the form $(28)(V=29.82$ $\mathrm{V})$.

data, and the results are quite good: the identified model is relevant and accurate, even in prediction situations.

The accuracy of the so-identified models suggests several applications in the future. Namely, a high precision tension reference can be built by controlling the micromirror around its semi-stable bifurcation point.

\section{REFERENCES}

G. Zhu, J. Penet, L. Saydy, Modeling and control of electrostatically actuated Mems in the presence of parasitics and parametric uncertainties, J. of Dyn. Syst., Meas. and Control, Vol. 129, pp 786-794, Nov. 2007.

K.-M. Liao, Y.-C. Wang, C.-H. Yeh and R. Chen, Closedloop adaptive control for torsional micromirrors. In ELFATATRY, AYMAN (editor): MOEMS and Miniaturized Systems IV, volume 5346 of Proceedings of SPIE, pages 184-192, Bellingham, WA, 2004. SPIE.

H. Sane, N. Yazdi, and C. Mastrangelo: Robust control of electrostatic torsional micromirrors using adaptive sliding-mode control. In Proceedings of SPIE - Photonics West 2005, volume 5719, pages 115-126, San Jose, CA, January 22-27, 2005.

M. F Daqaq, K. C. Reddy and A. H. Nayfeh, InputShaping Control of Nonlinear MEMS Proceedings of the IMAC-XXII Conference and Exposition on Structural Dynamics, Orlando, FL, 2006.
J. Bryzek, H. Abbott, A. Flannery, D. Cagle, J. Maitan, Control issues for MEMS Proceedings of the 42nd IEEE Conference on Decision and Control, vol.3, pp. 30393047 Vol.3, 9-12 Dec. 2003

L. Ljung, System identification - Theory for the user, Prentice Hall, 1999.

C. Casenave, E. Montseny, H. Camon, System Identification of Nonlinear Dynamic Models of Electrostatically Actuated MEMS, submitted to Control Engineering Practice, 2009.

H. Garnier, P. Young, Time-domain approaches to continuous-time model identification of dynamical systems from sampled data, Proceeding of the 2004 American Control Conference, Boston, June 30 - July 2, 2004.

H. Camon, C. Ganibal, N. Raphoz, M. Trzmiel, C. Pisella, C. Martinez, S. Valette, Solving functional reliability issue for an optical electrostatic switch, Microsystem Technologies, Vol. 14, N. 7, July 2008.

R.A. Adams, J.J.F. Fournier, Sobolev Spaces, Academic Press, 2003.

K. Yosida, Functional Analysis, Springer-Verlag, 1980.

L. Schumaker, Spline Functions: Basic Theory, Cambridge Mathematical Library, Sept. 2007.

A. Ben-Israel, T.N.E. Greville, Generalized inverses: theory and applications, Springer-Verlag, New York, USA, 2003.

H. Camon, F. Larnaudie, Fabrication, simulation and experiment of a rotating electrostatic silicon mirror with large angular deflection, 13th Int. Micro Electro Mechanical Systems (MEMS 2000), Miyazaki (Japan), Jan. 23-27, 2000, pp. 645-650. 